\title{
Automatic Concealed Heart Rate Detection from the BCG in Seated Position
}

\author{
P. S. Luna, R. Pallàs, Fellow Member, IEEE
}

\begin{abstract}
Continuous monitoring of health state is important in elder and chronically ill (but otherwise not critical) patients. Ideally, the patient should not be aware of being continuously monitored. This study describes an automatic and non-conscious heart rate monitoring system based on the ballistocardiogram (BCG). The bi-axial BCG can be recorded by two piezoelectric force sensors and high-gain amplifiers. Digital signal preprocessing has been implemented to increase the SNR and an algorithm to automatically detect the heart rate has been evaluated. The high gain required makes the system susceptible to forces other than those related to the cardiovascular system, such as those due to body movements. The sensitivity and positive predictive values obtained are $97.8 \%$ and $98.4 \%$ but if the records where the $\mathrm{BCG}$ is masked are discarded, the respective values increase to $99.2 \%$ and $99.5 \%$. These values are comparable to those typically obtained for the ECG.
\end{abstract}

Kwywords - Heart rate detection, non-conscious monitoring, ballistocardiogram

\section{INTRODUCCIÓN}

$\mathrm{E}_{\mathrm{a}}^{\mathrm{L}}$ L MONITOREO continuo del estado de salud contribuye a mejorar la calidad de vida de las personas y permite prevenir enfermedades o trastornos graves al detectar los síntomas tempranamente. En algunos grupos de la población, tales como las personas mayores o enfermos crónicos, es necesario seguir rutinariamente algunos parámetros fisiológicos que indiquen su estado de salud y mantener un registro de dichos parámetros. Esto, junto con otra información médica complementaria, permite establecer un punto de referencia y detectar posibles alteraciones para elaborar diagnósticos apropiados. Todas estas medidas, sin embargo, pierden interés por parte de los pacientes si les exigen trasladarse a un centro de salud o consultorio médico, a la vez que sus frecuentes visitas complican la gestión de los recursos sanitarios y a menudo repercuten seriamente en la situación económica y laboral de los pacientes y sus familiares.

Por lo anterior, y también porque algunos parámetros fisiológicos como la frecuencia cardiaca, la temperatura o la presión arterial se alteran por el nerviosismo de un examen rutinario [1]-[2], es preferible realizar el monitoreo del estado de salud en el hogar o en el trabajo, donde las personas desarrollan la mayor parte de sus actividades cotidianas, y

P. S. Luna, Facultad de Instrumentación Electrónica, Universidad Veracruzana, Xalapa, Veracruz, México, paluna@uv.mx

R. Pallàs, Grupo de Instrumentación, Sensores e Interfaces, Universitat Politècnica de Catalunya, Castelldefels (Barcelona), España, ramon.pallas@upc.edu

This work was performed in the Universitat Politèctica de Catalunya. hacerlo de una forma que pase desapercibida por el paciente para no alterar sus actividades. En este sentido, los dispositivos de monitoreo continuo que requieren colocar sensores en el cuerpo del paciente [3], [4], pueden hacerse incómodos y generar rechazo.

Los dispositivos de medida basados en interfaces con las cuales el usuario establece un contacto esporádico y breve durante el día [5], [6], permiten conocer el estado de salud de forma puntual. Esto puede ser suficiente para personas sanas o que no necesiten un seguimiento ininterrumpido. Con este objetivo se han propuesto sistemas para medir distintos parámetros cardiovasculares y respiratorios basados en interfaces donde se pasan largos periodos de tiempo: sillas, bancos, sillones, etc., (personas sentadas) y camas o interfaces similares (personas acostadas).

Las medidas en personas acostadas [7], [8] son indicadas cuando hay algún riesgo de muerte súbita durante el sueño o para monitorización continua en personas hospitalizadas. La monitorización durante el periodo diurno, en cambio, se puede hacer mientras una persona está sentada, pues en esta posición se pasa gran parte del tiempo. Si además los sensores no son evidentes en la interfaz (silla, banco, sillón, etc.) con la que entrará en contacto el paciente, las medidas pueden realizarse de forma completamente transparente, es decir, sin que note que esté siendo monitoreado, con lo cual se facilitan las medidas completamente no invasivas, y se podrá tener una mayor aproximación al estado de salud real de la persona.

En este trabajo se describe un sistema para medir de forma oculta la frecuencia cardiaca a partir del balistocardiograma, que es la señal debida a las fuerzas de reacción del cuerpo frente a la acción mecánica del corazón y al flujo sanguíneo por las principales arterias, medido en personas sentadas.

\section{El BALISTOCARDIOGRAMA}

La variación y el desplazamiento del volumen de sangre en el sistema cardiovascular durante el ciclo cardiaco provocan un desplazamiento del centro de gravedad del cuerpo [9]. Es decir, conforme la sangre fluye por la rama ascendente de la aorta y por las ramas carótidas, el cuerpo ejerce unas fuerzas de reacción en dirección caudal. Durante el recorrido de la sangre por la aorta descendente y sus ramificaciones, el cuerpo reacciona en dirección cefálica. Estas fuerzas de reacción inducen en el cuerpo unos desplazamientos en tres direcciones: cefálico-caudal (longitudinal o vertical), anteroposterior (transversal u horizontal) y lateral (izquierdaderecha) [10]. El registro de estas fuerzas de reacción o de los movimientos corporales que producen se denomina balistocardiograma (BCG). 
La Fig. 1 representa la forma de onda típica del BCG registrado en dirección vertical en una persona sentada. La flecha discontinua indica la posición temporal de la onda $\mathrm{R}$ del electrocardiograma (ECG) medido simultáneamente. Cada una de las ondas del BCG ha sido nombrada con letras sucesivas desde la $\mathrm{G}$ hasta la $\mathrm{O}$. En algunas situaciones, sobre todo con frecuencia cardiaca elevada, la última oscilación de un ciclo cardiaco (onda $\mathrm{O}$ ) se solapa con la primera del siguiente (onda $G$ ), por lo que son difíciles de identificar.

La Fig. 2 muestra una concepción cualitativa de la generación del BCG, desde el origen de los eventos mecánicos hasta su registro por un sistema electrónico. El ECG, cuya aparición depende del Sistema Nervioso Autónomo (SNA) provoca una serie de eventos mecánicos que son filtrados por el medio de transmisión (músculos, órganos internos y estructura ósea) y por la interfaz mecánica en contacto con el cuerpo. La señal mecánica se detectaría con un transductor de fuerza o movimiento a señal eléctrica, la cual se filtra nuevamente por el sistema de registro (instrumentación electrónica), obteniéndose con esto el BCG.

Debido a los diferentes medios y procesos que afectan al $\mathrm{BCG}$, su forma de onda varía notablemente de un sujeto a otro, y en ocasiones incluso en un mismo sujeto de un latido al siguiente [11]-[13]. Esto, junto con el desarrollo de métodos más fiables como el cateterismo y la ultrasonografía, llevó hace años al abandono de esta técnica. Sin embargo, los recursos técnicos actuales permiten obtener fácilmente esta señal que contiene información potencialmente importante sobre la actividad mecánica del sistema cardiovascular, aunque el origen de las ondas registradas aún es mucho menos conocido que el del ECG. No obstante, muchos autores coinciden en que las ondas I, J y K son debidas a la expulsión de sangre por los ventrículos y a su recorrido por las ramas principales de la aorta. Esto, junto con el hecho de que estas ondas son las oscilaciones de mayor amplitud del BCG, es una razón por la que en estudios recientes [5], [6] se haga énfasis en la detección de estas ondas como un método para medir la frecuencia cardiaca, que es también el método adoptado en este trabajo.

A mediados del siglo XX el $\mathrm{BCG}$ se registraba en la dirección cefálico-caudal en personas acostadas. Actualmente

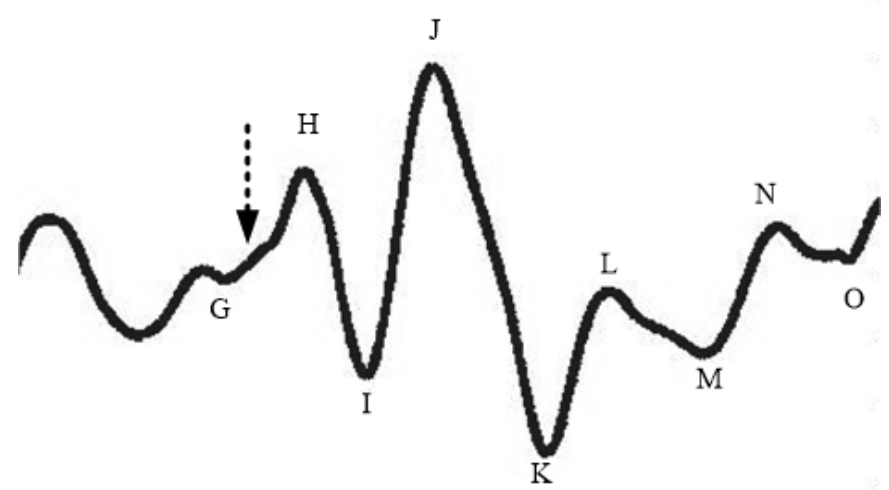

Figura 1. Balistocardiograma vertical registrado en una persona sentada. La flecha discontinua en el BCG indica la ubicación temporal de la onda R del ECG.

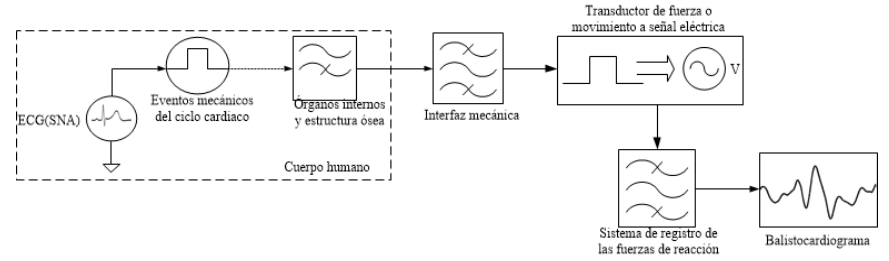

Figura 2. Concepción cualitativa del origen y registro del BCG.

se mide comúnmente en la misma dirección pero en personas de pie sobre alguna plataforma [5], [6]. Algunos trabajos recientes sobre el BCG en personas sentadas [14], [15] lo han medido en la dirección vertical (en el asiento) y además en la transversal (en el respaldo), lo cual puede aportar más información que el análisis de una sola componente del BCG.

Dado que la morfología del BCG es muy variable de un ciclo cardiaco a otro, la identificación automática de latidos se complica si no se utiliza alguna otra señal como referencia. En este trabajo se obtiene la frecuencia cardiaca de forma oculta y automática a partir del BCG medido en dos direcciones (vertical y transversal) para evaluar la factibilidad de utilizar ambas componentes como información complementaria.

\section{MÉTODO DE MEDIDA DEL BCG}

\section{A. Sistema de registro de las señales de fuerza}

Las fuerzas de reacción del cuerpo deforman las superficies que estén en contacto con él. En personas sentadas, estas superficies son el respaldo y el asiento. Para medir estas deformaciones en una silla convencional de oficina, se han fijado con cinta adhesiva sendos sensores piezoeléctricos en la cara posterior del respaldo, para obtener el BCG transversal, e inferior del asiento, para medir el BCG vertical. El espesor del sensor (inferior a $0,5 \mathrm{~mm}$ ) hace que pase desapercibido una vez que se ha cubierto con la tapicería de la silla.

Las variaciones de la carga eléctrica a la salida del sensor se transforman en variaciones de tensión con un amplificador de carga; posteriormente, esta señal se filtra pasa banda para eliminar la tensión de desplazamiento (offset) y reducir el ancho de banda de ruido, y se digitaliza para su procesamiento en un ordenador personal. La Fig. 3 muestra el diagrama de bloques del sistema de medida utilizado.

En condiciones normales, el corazón de una persona sana en reposo expulsa entre $70 \mathrm{~mL}$ y $80 \mathrm{~mL}$ de sangre en cada latido a una velocidad media $v=0,33 \mathrm{~m} / \mathrm{s}$ [16]. Considerando que la densidad de la sangre de una persona sentada es $\rho=1043 \mathrm{~kg} / \mathrm{m}^{3}$ [17], la masa de sangre expulsada en cada latido por una persona sana en reposo es de unos $73 \mathrm{~g} \mathrm{y}$ suponiendo una duración de la sístole de 0,6 s [16], la fuerza instantánea $F$ aproximada ejercida por el ventrículo izquierdo puede estimarse a partir de la ecuación 1:

$$
F=m a=40 \times 10^{-3} \mathrm{~N} .
$$

A esta fuerza hay que añadir la del ventrículo derecho, la debida a la elasticidad de la aorta y las arterias principales, y también la de la respiración y otros movimientos corporales voluntarios o no. Dado que estos últimos tienen mayor amplitud que los primeros, serán los que definan la ganancia 


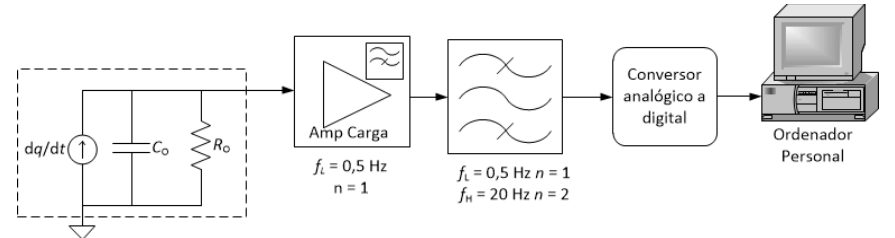

Figura 3. Sistema de medida de fuerza con un sensor piezoeléctrico.

máxima que puede tener el sistema de registro para evitar su saturación.

El ancho de banda necesario para medir el BCG es desde $0,01 \mathrm{~Hz}$ hasta $40 \mathrm{~Hz}$ [18]; sin embargo, la mayor concentración de energía está por debajo de los $20 \mathrm{~Hz}$ [14] por lo que comúnmente se utiliza este valor como límite superior de la banda de paso, $f_{\mathrm{H}}$. La frecuencia inferior $f_{\mathrm{L}}$ depende del interés en el contenido de bajas frecuencias del BCG y de las interferencias mecánicas presentes (oscilaciones y vibraciones externas) en el sitio de la medida. En este trabajo se ha elegido $f_{\mathrm{L}}=0,5 \mathrm{~Hz}$ y $f_{\mathrm{H}}=20 \mathrm{~Hz}$. De esta forma se atenúan las componentes respiratorias sin alterar la información de la frecuencia cardiaca, suponiendo que el monitoreo se realizará en personas en reposo, y se reducen las interferencias eléctricas de alta frecuencia que pudieran acoplarse a la señal.

El filtro pasa altas se ha obtenido con la respuesta del amplificador de carga y con un filtro pasivo de primer orden a la salida de éste, obteniendo así una respuesta total de segundo orden a la misma frecuencia de corte. El ancho de banda se ha limitado con un filtro activo $(n=2)$ con respuesta Butterworth. El condensador que define la ganancia de cada amplificador se ha establecido como $C=100 \mathrm{pF}$ para el BCG vertical y $C=1 \mathrm{nF}$ para el transversal. La resistencia de polarización del amplificador de carga, en paralelo con este condensador consistía en una red en $\mathrm{T}$ de resistores de bajo costo.

\section{B. Estimación del ruido del amplificador de carga}

Para medir el ruido en el amplificador de carga, se ha conectado su entrada a la masa de señal $(0 \mathrm{~V})$ mediante un condensador de $10 \mathrm{nF}$, una capacidad similar a la capacidad del sensor piezoeléctrico utilizado [19]. Para reducir las interferencias electromagnéticas, el circuito se ha alimentado con baterías $( \pm 6 \mathrm{~V})$ y se ha colocado dentro de una caja metálica conectada también a $0 \mathrm{~V}$. La tensión de salida se ha amplificado con la etapa de entrada de un osciloscopio de alta ganancia (Tektronix 7A22 conectado a 7904A) y la salida se digitalizaba con un registrador gráfico de 12 bit (Yokogawa DL750) tomando 5000 muestras cada segundo. Se han realizado cinco registros con un ancho de banda de $0.2 \mathrm{~Hz}$ a $20 \mathrm{~Hz}$ y en cada uno se ha calculado la desviación estándar. La tensión de ruido en valor eficaz se ha estimado como el valor promedio de la desviación estándar de los cinco registros.

\section{Comportamiento dinámico de la interfaz mecánica}

En los trabajos recientes sobre la medida del BCG en personas sentadas, no se evalúa la respuesta dinámica de la interfaz mecánica, y a veces ni tan siquiera se menciona. Sin embargo, si se pretende obtener información adicional a la frecuencia cardíaca, es importante considerarla para garantizar que no alterará radicalmente la forma de onda de los impulsos mecánicos registrados [13]. Para ello es necesario que la respuesta sea plana en la banda de frecuencias de interés, y el desfase proporcional a la frecuencia.

En trabajos realizados en personas de pie o acostadas, la respuesta dinámica de las interfaces se ha medido aplicando un impulso mecánico y ajustando la respuesta de cada interfaz a un modelo matemático predefinido [5] o mediante análisis espectral [20]. Sin embargo, la respuesta dinámica de una silla ante un impulso mecánico depende totalmente de cómo esté construida, por lo que los resultados obtenidos para un modelo concreto difícilmente se podrán generalizar.

Dado que el objetivo aquí es detectar sólo la frecuencia cardíaca, basta comprobar que el sistema no atenúa notablemente las vibraciones de origen cardíaco. Para ello, se ha golpeado el asiento y el respaldo con una masa de 57,5 g cuando había distintas masas colocadas sobre el asiento y posteriormente se ha obtenido su espectro de frecuencias utilizando la función FFT de Matlab®.

La respuesta de la silla se ha medido con un sensor piezoeléctrico en la banda de $0,1 \mathrm{~Hz}$ a $10 \mathrm{kHz}$. La tensión a la salida del filtro pasa banda se ha registrado con una resolución de 12 bit y tasa de muestreo de 100000 muestras por segundo.

\section{Pre-procesamiento digital del BCG}

Además de la respiración y otros movimientos corporales, durante el registro del BCG se pueden presentar algunas fluctuaciones debidas a vibraciones externas que degradan la Relación Señal a Ruido (SNR) y dificultan la ubicación automática de latidos. Por este motivo, antes de procesar la señal en busca de la actividad cardiaca y respiratoria, es necesario mejorar la SNR con técnicas digitales que faciliten esta tarea. En primer lugar se atenúa la componente de la respiración filtrando la señal en la banda de $0,5 \mathrm{~Hz}$ a $20 \mathrm{~Hz}$ con un filtro digital de orden 2 y con una respuesta lineal en fase. Posteriormente se eliminan las fluctuaciones de la línea base utilizando un algoritmo comúnmente utilizado en electrocardiografía que consiste en estimar la componente de baja frecuencia de las oscilaciones y restarla de la señal original [21]. Una vez hecho esto se realzan las oscilaciones debidas a la actividad cardiaca filtrando pasa banda el BCG con frecuencias de corte de $3 \mathrm{~Hz}$ y $10 \mathrm{~Hz}$. Posteriormente, se eleva el BCG a la tercera potencia, para conservar la polaridad de la señal y realzar las oscilaciones principales [22], aplicando ventanas temporales con duración de $1,2 \mathrm{~s}$, que es suficientemente larga para incluir frecuencias cardiacas bajas.

Una vez que se han realzado las oscilaciones principales de cada ciclo cardiaco, se calcula la derivada de esta señal y se localizan las oscilaciones que superen un cierto umbral preestablecido (entre 0,15 y 0,25 del valor máximo del registro). Las oscilaciones encontradas se consideran correspondientes a la onda $\mathrm{J}$ del BCG, que es habitualmente la de mayor amplitud. Para evitar Falsos Positivos (FP), se avanza temporalmente la señal un cierto valor antes de 
comenzar la búsqueda de la siguiente onda J. El valor de este desplazamiento temporal se ha definido como el $80 \%$ del valor medio de las separaciones entre las ondas $\mathrm{J}(\mathrm{mJ}-\mathrm{J})$ que ya han sido detectadas.

Para localizar Falsos Negativos (FN) una vez que se ha analizado todo el registro, se buscan dos oscilaciones que estén separadas más del $120 \%$ de mJ-J. Si se encuentran, se repite la búsqueda de una oscilación grande entre estas dos ondas J. Por el contrario, si la separación es inferior al $50 \%$ de mJ-J, se identifica como FP y se descarta la oscilación cuya separación es inferior al umbral.

El pre-procesamiento digital se ha implementado en Matlab ${ }^{\circledR}$ a partir del BCG filtrado en la banda de $0,5 \mathrm{~Hz}$ a $20 \mathrm{~Hz}\left(\mathrm{BCG}_{\mathrm{FPB}}\right)$.

\section{RESULTADOS Y DISCUSIÓN}

\section{A. Estimación de ruido del amplificador de carga}

La tensión de ruido de pico a pico respectivas del amplificador de BCG transversal y vertical, referidas a la salida se indican en las ecuaciones 2 y 3 :

$$
\begin{aligned}
& E_{\text {noT }}=191 \mu \mathrm{V} \\
& E_{\text {noV }}=1,9 \mathrm{mV}
\end{aligned}
$$

donde se ha considerado un Factor de Cresta $\mathrm{CF}=6,6$, que corresponde a una probabilidad de que la tensión de pico a pico exceda el valor calculado sólo un $0,1 \%$ de la duración del registro [23].

A partir de las especificaciones del sensor y de la ganancia utilizada, la fuerza máxima respectiva ejercida sobre el sensor colocado en el respaldo y en el asiento está dada por los valores mostrados en las ecuaciones 4 y 5 :

$$
\begin{aligned}
& F_{3 \mathrm{R}}=\frac{Q_{\mathrm{nT}}}{d_{31}}=8,30 \mathrm{mN} \\
& F_{3 \mathrm{~A}}=\frac{Q_{\mathrm{nV}}}{d_{31}}=8,26 \mathrm{mN}
\end{aligned}
$$

Estos valores representan un $20 \%$ de los $40 \mathrm{mN}$ estimados en (1).

\section{B. Comportamiento dinámico de la interfaz mecánica}

La Fig. 4 muestra la respuesta en frecuencia del asiento cargado con distintas masas. Se observa que para masas superiores a $32 \mathrm{~kg}$ la principal componente de frecuencias permanece constante alrededor de unos $8,5 \mathrm{~Hz}$.

Para evaluar la respuesta dinámica del respaldo se ha aplicado un impulso mecánico en su parte frontal dejando caer una pelota de 57,5 g con un movimiento pendular desde una altura vertical de $54 \mathrm{~cm}$. Este experimento se ha repetido cargando al asiento con distintas masas. El volumen de los objetos sobre el asiento (paquetes de folios), para lograr masas superiores a $40 \mathrm{~kg}$, dificultaba el trayecto de la pelota, por lo que se ha evaluado la respuesta dinámica sólo hasta $40 \mathrm{~kg}$.

La Fig. 5 muestra el espectro de frecuencias de la respuesta en el tiempo del respaldo con distintas masas en el asiento. Se observa que presenta una frecuencia de resonancia superior a

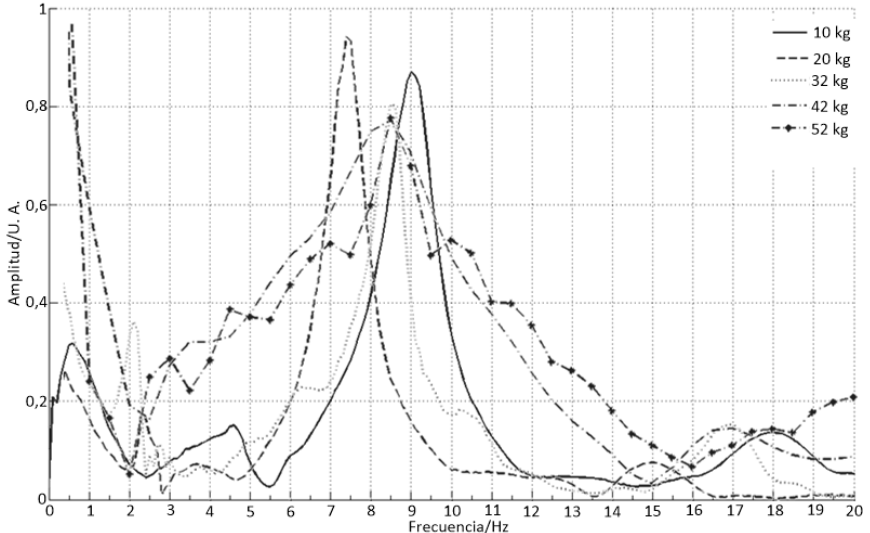

Figura 4. Respuesta en frecuencia del asiento ante un impulso mecánico con distintas masas sobre él.

la del asiento y que se desplaza ligeramente hacia la derecha conforme aumenta la masa depositada en el asiento de la silla. Para una masa de $12,5 \mathrm{~kg}, f_{\mathrm{n}}=28,6 \mathrm{~Hz}$ y para $40 \mathrm{~kg}$, $f_{\mathrm{n}}=29,4 \mathrm{~Hz}$.

Esta evaluación es únicamente orientativa porque la distribución de la masa sobre el asiento puede alterar la respuesta en el respaldo, y en un sistema de medida del BCG, el impulso mecánico (latido cardiaco) se transmitirá por un medio que puede cambiar la respuesta de todo el sistema (Fig. 2). En cualquier caso, la frecuencia de resonancia tan alta obtenida para esta interfaz permite suponer que las componentes fundamentales del BCG no quedarán alteradas.

\section{Pre-procesamiento digital del $B C G$}

La Fig. 6 muestra un segmento del registro del BCG transversal, medido en el respaldo (gráfica superior), y el BCG vertical, medido en el asiento (gráfica inferior), con la corrección de la línea base. Las oscilaciones de alta frecuencia del BCG vertical pueden ser debidas a movimientos corporales involuntarios que, al ser rápidos, presentan unas componentes de frecuencia altas, por lo que quedan fuera del ancho de banda del filtro digital empleado y de la frecuencia de muestreo reducida.

\section{Detección automática de la frecuencia cardiaca}

Se ha registrado el BCG en dos direcciones en trece voluntarios. Como señal de referencia se ha medido el

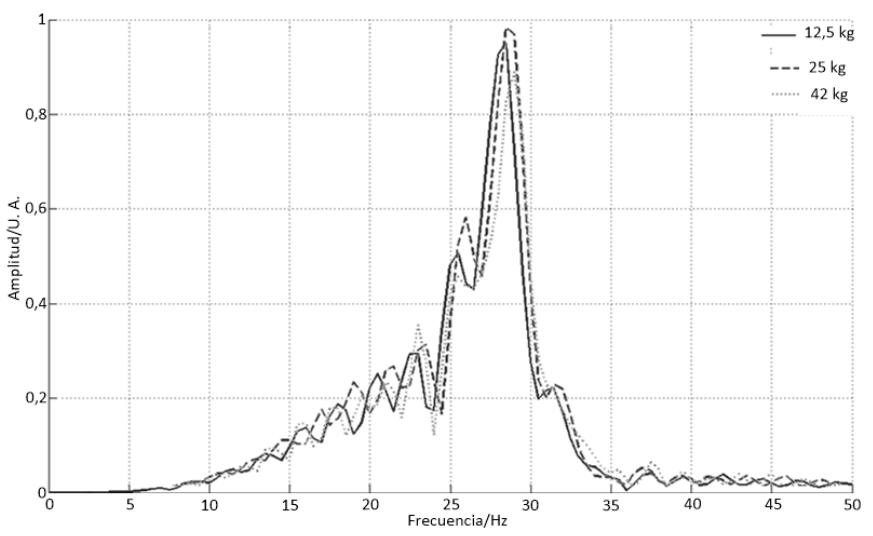

Figura 5. Amplitud de la respuesta del respaldo en el dominio de la frecuencia ante un impulso mecánico. 


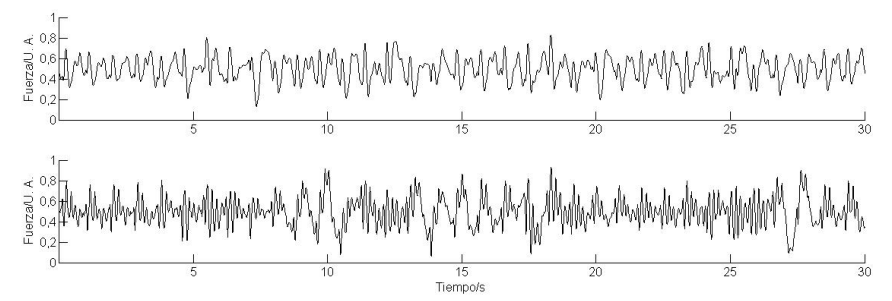

Figura 6. BCG transversal, BCG vertical después de eliminar las fluctuaciones de la línea base y movimientos debidos a la respiración.

Fotopletismograma (PPG) en el dedo índice de la mano izquierda. La duración de los registros ha sido de $60 \mathrm{~s}$ y las señales se han adquirido con una tarjeta de adquisición de datos (Eagle uDAQ) de 12 bit controlada por un ordenador (portátil) personal, tomando 100 muestras cada segundo.

Para evaluar los resultados del algoritmo se ha calculado la Sensibilidad $(S)$ y la Predictividad Positiva $(P P)$, definidas por la normativa AAMI [24] para evaluar algoritmos de detección del ritmo cardiaco, como

$$
\begin{aligned}
& S=\frac{\mathrm{REF}}{\mathrm{REF}+\mathrm{FN}} \\
& P P=\frac{\mathrm{REF}}{\mathrm{REF}+\mathrm{FP}}
\end{aligned}
$$

donde REF es el número de latidos en la señal de referencia, el cual se ha considerado que es correcto, condición que se ha cumplido en todos los casos.

La Tabla 1 muestra los latidos encontrados a partir de la señal de referencia, del BCG transversal (BCGT) y del BCG vertical (BCGV), y los falsos positivos y negativos para cada registro. Con los datos medidos se tiene, para el BCG vertical, una sensibilidad y predicción positiva de

y para el BCG transversal:

$$
S_{\mathrm{V}}=97,8 \% ; \quad P P_{\mathrm{V}}=98,4 \%,
$$

$$
S_{\mathrm{T}}=97,8 \% ; \quad P P_{\mathrm{T}}=98,4 \% .
$$

Estos valores, relativamente bajos (los valores típicos para un algoritmo de detección ECG son $\mathrm{S}=99,7 \%$ y $\mathrm{PP}=99,8 \%$ [25]) se deben a la incertidumbre en la detección de los latidos de los voluntarios 3, 7 y 13, los cuales han presentado unas formas de onda peculiares en al menos una señal de BCG. En estos casos la actividad cardiaca no se distingue bien debido a que también se han registrado otras oscilaciones con componentes de frecuencia similares a los del latido cardiaco. En otras sesiones de experimentos, donde se ha medido el BCG vertical de pie, la forma del BCG de estas personas también era atípica.

$\mathrm{Si}$ se omiten los registros de los tres voluntarios que presentaron formas de onda atípicas, se tiene entonces, para la dirección vertical:

y para el BCG transversal:

$$
S_{\mathrm{V}}=99,1 \% ; \quad P P_{\mathrm{V}}=99,3 \%,
$$

$$
S_{\mathrm{T}}=99,2 \% ; \quad P P_{\mathrm{T}}=99,5 \% \text {. }
$$

Estos resultados son bastante más parecidos a los resultados de los algoritmos tradicionales para la detección en línea de los latidos a partir del ECG y el procesamiento es sencillo comparado con utilizar transformada wavelet o redes

\begin{tabular}{|c|c|c|c|c|c|c|c|}
\hline$n$ & REF & BCGT & FP & FN & BCGV & FP & $\mathrm{FN}$ \\
\hline 1 & 76 & 78 & 1 & 0 & 77 & 0 & 0 \\
\hline 2 & 53 & 54 & 0 & 0 & 54 & 0 & 0 \\
\hline 3 & 71 & 72 & 9 & 9 & 69 & 5 & 8 \\
\hline 4 & 61 & 61 & 1 & 1 & 62 & 1 & 2 \\
\hline 5 & 71 & 72 & 0 & 0 & 70 & 0 & 2 \\
\hline 6 & 53 & 55 & 1 & 2 & 55 & 3 & 2 \\
\hline 7 & 67 & 67 & 3 & 3 & 69 & 4 & 4 \\
\hline 8 & 78 & 79 & 0 & 0 & 78 & 1 & 1 \\
\hline 9 & 75 & 76 & 2 & 3 & 75 & 0 & 0 \\
\hline 10 & 61 & 62 & 2 & 1 & 61 & 0 & 1 \\
\hline 11 & 61 & 62 & 0 & 0 & 62 & 0 & 0 \\
\hline 12 & 73 & 74 & 2 & 2 & 74 & 3 & 3 \\
\hline \multirow[t]{2}{*}{13} & 62 & 57 & 14 & 19 & 61 & 8 & 10 \\
\hline & 2526 & 1688 & 34 & 50 & 1693 & 34 & 48 \\
\hline
\end{tabular}
neuronales, como comúnmente se reporta en la bibliografía.
TABLA I. NÚMERO DE LATIDOS ENCONTRADOS A PARTIR DE LA SEÑAL DE REFERENCIA (REF), DEL BCG TRANSVERSAL (BCGT) Y DEL BCG VERTICAL (BCGV) Y FALSOS POSITIVOS (FP) Y NEGATIVOS (FN) EN REGISTROS DE $60 \mathrm{~s}$ DE DURACIÓN.

Con estos resultados se considera que es posible medir la frecuencia cardiaca de forma oculta en personas sanas sentadas midiendo el BCG en dirección transversal o vertical. Aunque no para todos los casos sea posible medir con precisión el número de latidos por minuto, la información extraída durante los periodos en que hay poco movimiento se podría utilizar para estimar la evolución de la actividad cardiaca a lo largo del tiempo, puesto que los resultados presentan una sensibilidad superior al $99 \%$.

En algunos casos se ha observado que la forma de la señal registrada no presenta una información clara sobre la actividad cardiaca. Utilizando métodos y/o algoritmos más complejos, podría mejorarse la resolución en la ubicación de los ciclos cardiacos sin otra señal como referencia. No obstante, esto complicaría el procesamiento y elevaría el coste del sistema, que es algo que se ha intentado evitar en el trabajo aquí presentado, pero podría ser una opción válida en determinadas aplicaciones.

\section{CONCLUSIONES}

Se ha demostrado la posibilidad de medir de forma oculta la actividad cardiaca en personas sentadas a partir del BCG con un algoritmo sencillo, de bajo costo computacional y sin utilizar ninguna otra señal como referencia, a diferencia de otros trabajos reportados en la bibliografía donde se emplean herramientas que complican su implementación [7] o donde se depende de otra señal cardiaca, normalmente el ECG [6].

Por la baja amplitud del BCG (aproximadamente $40 \mathrm{mN}$ ), es necesario amplificar mucho la señal y esto conlleva interferencias debidas a los movimientos, voluntarios o no, del sujeto bajo observación e incluso a las vibraciones presentes en el sitio de la medida, y cuyo ancho de banda caiga dentro del rango de frecuencias detectadas por el sistema. Para reducir estas vibraciones mecánicas externas, siempre que no saturen el amplificador, se ha implementado una técnica simple de procesamiento con la cual se obtiene una señal más limpia para buscar la actividad cardiaca. Con las señales 
limpias, se ha demostrado que es posible detectar los latidos cardiacos con una sensibilidad superior al $99 \%$ y una predictividad positiva mayor al $99.5 \%$ en sujetos que presenten señales con formas típicas. Sin embargo, incluso en personas sanas sin ninguna patología conocida, aparecen formas de onda irregulares.

\section{ACKNOWLEDGMENT}

This work has been funded by the Spanish Ministry of Science and Innovation under contract TEC2009-13022 and by the European Regional Development Fund.

\section{REFERENCES}

[1] G. Mancia, G. Parati, G. Pomidossi, G. Grassi, R. Casadei and A. Zanchetti, "Alerting reaction and rise in blood pressure during measurement by physician and nurse", Hypertension, vol. 9, no. 2, pp. 209-215, 1987.

[2] P. Owens, N. Atkins and E. O'Brien, "Diagnosis of white coat hypertension by ambulatory blood pressure monitoring", Hypertension, vol. 34, no. 2, pp. 267-272, 1999.

[3] L. P. Córdoba, M. A. G. López, C. B. Goy and M. C. Herrera, "Design and Evaluation of Pressure and Electrocardiographic Modules Embedded in a Cardiac Hemodynamic Monitor", Latin America Transactions, IEEE, vol.11, no.1, pp.101-107, 2013.

[4] J. P. Tello, O. Manjarres, M. Quijano, A. Blanco, F. Varona, M. Manrique, "Remote Monitoring System of ECG and Human Body Temperature Signals", Latin America Transactions, IEEE, vol.11, no.1, pp.314-318, 2013

[5] R. Gonzalez-Landaeta, O. Casas and R. Pallas-Areny, "Heart rate detection from an electronic weighing scale", Physiol Meas, vol. 29, no. 8, pp. 979-88, 2008.

[6] O. Inan, M. Etemadi, R. Wiard, L. Giovangrandi and G. Kovacs, "Robust ballistocardiogram acquisition for home monitoring", Physiol Meas, vol. 30, no. 2, pp. 169-185, 2009.

[7] Y. Chee, J. Han, J. Youn and K. Park, "Air mattress sensor system with balancing tube for unconstrained measurement of respiration and heart beat movements", Physiol Meas, vol. 26, no. 4, pp. 413-422, 2005

[8] W. Chen, X. Zhu, T. Nemoto, K. Kitamura, K. Sugitani and D. Wei, "Unconstrained monitoring of long-term heart and breath rates during sleep", Physiol Meas, vol. 29, no. 2, pp. N1-N10, 2008.

[9] A. Noordergraaf and C. Heynekamp, "Genesis of displacement of the human longitudinal ballistocardiogram from the changing blood distribution", Am J Cardiol, vol. 2, no. 6, pp. 748-756, 1958.

[10] R. Soames and J. Atha, "Three-dimensional ballistocardiographic responses to changes of posture", Clin Phys Physiol M, vol. 3, no. 3, pp. 169-177, 1982.

[11] P. Pollock, "Ballistocardiography: a clinical review", Can Med Assoc J, vol. 76, no. 9, pp. 778-783, 1957.

[12] R. Gubner, M. Rodstein and H. Ungerleider, "Ballistocardiography: An appraisal of technic, physiologic principles, and clinical value", Circulation, vol. 7, no. 2, pp. 268-286, 1953.

[13] M. Rappaport, H. Sprague, and W. Thompson, "Ballistocardiography: I. Physical Considerations”, Circulation, vol. 7, no. 2, pp. 229-246, 1953.

[14] S. Junnila, A. Akhbardeh, L. Barna, I. Defee and A. Varri, "A wireless ballistocardiographic chair", in Proc. 28th Int. Conf. of the IEEE EMBS, New York, NY, pp. 5932-5935, 2006.

[15] P. S. Luna-Lozano, and C. Alvarado-Serrano, "Time and amplitude relationships of the ballistocardiogram in vertical and horizontal direction", in Proc 9th Int. Conf. of Electrical Engineering, Computing Science and Automatic Control, Mexico City, pp 1-6, 2012.

[16] A. Guyton, and J. Hall, 2000, Textbook of medical physiology, 10th edition, W. B. Saunders Co., Philadelphia Pa.

[17] H. Hinghofer-Szalkay, and J. Greenleaf, "Continuous monitoring of blood volume changes in humans", J Appl Physiol, vol. 63, no. 3, pp. 1003-1007, 1987.

[18] W. Olson, 2010, "Basic concepts of medical instrumentation" in Medical instrumentation: application and design, edited by J.G. Webster, 4th edition, John Wiley \& Sons, Inc., Hoboken, pp. 1-44.
[19] Measurement Specialties Inc., 1999, Piezo film sensors technical manual.

[20] R. Pallàs-Areny and J. Webster, 2001, Sensors and Signal Conditioning, 2nd edition, John Wiley \& Sons, Inc., New York.

[21] L. Sörnmo and P. Laguna, 2006, "Electrocardiogram (ECG) signal processing" in Wiley Encyclopedia of Biomedical Engineering, edited by M. Akay, John Wiley and Sons, Inc., pp. 1298-1313.

[22] P. Smrcka, M. Jirina, Z. Trefny and K. Hana, "New methods for precise detection of systolic complexes in the signal acquired from quantitative seismocardiograph", in Proc. of the IEEE Int. Workshop on Intelligent Signal Processing, pp. 375-380, 2005.

[23] Pallàs-Areny and J. Webster, 1999, Analog Signal Processing, John Wiley \& Sons, Inc., New York.

[24] AAMI Association for the Advancement of Medical Instrumentation 1998, ANSI/AAMI EC57:1998 -Testing and reporting performance results of cardiac rhythm and ST segment measurement algorithms, (R) 2008, American National Standards Institute, Inc., Arlington, VA.

[25] P. Hamilton and W. Tompkins, "Quantitative investigation of QRS detection rules using the MIT/BIH arrhythmia database", IEEE T BioMed Eng, vol. BME-33, no. 12, pp. 1157-1165, 1986.

Pablo S. Luna-Lozano Received the Engineer in Electronics Instrumentation degree from the University of Veracruz (UV) in 2002, the MSc degree from

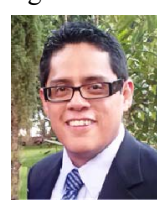
the CINVESTAV-IPN in 2003 and the $\mathrm{PhD}$ in the Universitat Politècnica de Catalunya (UPC) in 2011.

$\mathrm{He}$ is with the UV since 2012 teaching courses of biomedical and electronic instrumentation. His research areas include analog signal processing and non-conscious monitoring of physiological parameters.

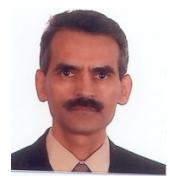

Ramon Pallàs-Areny received the Ingeniero Industrial and Doctor Ingeniero Industrial degrees from the Universitat Politècnica de Catalunya (BarcelonaTech, UPC), Barcelona, Spain, in 1975 and 1982, respectively. Since 1989 he is a Full Professor of Electronic Engineering at the same University, and teaches courses in electronic instrumentation. In 1989 and 1990 he was a visiting Fulbright Scholar and in 1997 and 1998 he was an Honorary Fellow at the University of Wisconsin, Madison. His research includes instrumentation methods and sensors based on electrical impedance measurements, noninvasive physiological measurements, autonomous sensors and sensor interfaces. He is the author of six books, the leading author of five books and coauthor of three books on instrumentation in Spanish and Catalan, with several editions. He is also coauthor of six books in English, one of them translated into Simplified Chinese. He has also authored 15 book chapters and coauthored 2. He has supervised 23 Ph.D. theses and published about 80 papers in peer-reviewed journals and more than 140 communications in international conferences. He was a recipient, with John G. Webster, of the 1991 Andrew R. Chi Prize Paper Award from the Instrumentation and Measurement Society (IEEE). In 1998 he became a Fellow of the IEEE. In 2000 he received the Award for Quality in Teaching granted by the Board of Trustees of the UPC, and in 2002 the Narcís Monturiol Medal from the Autonomous Government of Catalonia for his academic work and scientific contributions to electronic and medical instrumentation. 